\title{
PICO Questions and DELPHI Methodology for the Management of Venous Thromboembolism Associated with COVID-19
}

\author{
Antoni Riera-Mestre ${ }^{1,2, *(\mathbb{D})}$, Luis Jara-Palomares ${ }^{3,4}{ }^{(\mathbb{D}}$, Ramón Lecumberri ${ }^{5,6}{ }^{\mathbb{D}}$, Javier Trujillo-Santos ${ }^{7,8} \mathbb{D}$, \\ Enric Grau ${ }^{9}$, Angeles Blanco-Molina ${ }^{10}$, Ana Piera Carbonell ${ }^{11}{ }^{1}$, Sonia Jiménez ${ }^{12}$, Manuel Frías Vargas ${ }^{13}$, \\ Mari Paz Fuset ${ }^{14}$, Sergi Bellmunt-Montoya ${ }^{15,16,17}$ (D), Manuel Monreal ${ }^{8,18}\left(\mathbb{D}\right.$, David Jiménez ${ }^{4,19,20}$ (D) \\ and on behalf of the COVILAX Project ${ }^{+}$
}

check for updates

Citation: Riera-Mestre, A.;

Jara-Palomares, L.; Lecumberri, R.;

Trujillo-Santos, J.; Grau, E.;

Blanco-Molina, A.; Piera Carbonell,

A.; Jiménez, S.; Frías Vargas, M.;

Fuset, M.P.; et al. PICO Questions and

DELPHI Methodology for the

Management of Venous

Thromboembolism Associated with COVID-19. Viruses 2021, 13, 2128. https://doi.org/10.3390/v13112128

\section{Academic Editors:}

Giuseppe Camporese and

Pierpaolo Di Micco

Received: 22 September 2021

Accepted: 19 October 2021

Published: 22 October 2021

Publisher's Note: MDPI stays neutral with regard to jurisdictional claims in published maps and institutional affiliations.

Copyright: (c) 2021 by the authors. Licensee MDPI, Basel, Switzerland. This article is an open access article distributed under the terms and conditions of the Creative Commons Attribution (CC BY) license (https:/ / creativecommons.org/licenses/by/ $4.0 /)$.
1 Internal Medicine Department, Hospital Universitari de Bellvitge, Bellvitge Biomedical Research Institute (IDIBELL), 08907 Barcelona, Spain

2 Faculty of Medicine and Health Sciences, Universitat de Barcelona, 08907 Barcelona, Spain

3 Medical-Surgical Unit for Respiratory Diseases, Virgen del Rocío University Hospital, 28029 Sevilla, Spain; luisoneumo@hotmail.com

4 Center for Biomedical Research in Respiratory Diseases Network (CIBERES), Instituto de Salud Carlos III, 28029 Madrid, Spain; djimenez.hrc@gmail.com

5 Hematology Department, Clínica Universidad de Navarra, 31008 Pamplona, Spain; rlecumber@unav.es

6 Center for Biomedical Research Network on Cardiovascular Diseases (CIBERCV), Instituto de Salud Carlos III, 28029 Madrid, Spain

7 Internal Medicine Department, Hospital General Universitario Santa Lucía, 30204 Cartagena, Spain; javier.trujillosantos@gmail.com

8 Faculty of Health Sciences, Universidad Católica San Antonio de Murcia (UCAM), 30107 Murcia, Spain; mmonreal.germanstrias@gencat.cat

9 Hematology Department, Lluis Alcanyis de Xativa Hospital, 46800 Valencia, Spain; grau_enr@gva.es

10 Internal Medicine Unit, Hospital Universitario Reina Sofía, 10004 Córdoba, Spain; mablancom@telefonica.net

11 Luanco Health Center, 33440 Gozón, Spain; apiecar@gmail.com

12 Emergency Area, Hospital Clínic, Institut d'Investigacions Biomèdiques August Pi i Sunyer (IDIBAPS), 08036 Barcelona, Spain; sjimenez131707@gmail.com

13 Comillas Health Center, 28019 Madrid, Spain; manueldejesus.frias@salud.madrid.org

14 Intensive Medicine Department, Hospital Universitari de Bellvitge, Bellvitge Biomedical Research Institute (IDIBELL), 08907 Barcelona, Spain; mfuset@bellvitgehospital.cat

15 Angiology and Vascular Surgery Department, Hospital Universitari Vall d'Hebron, 08035 Barcelona, Spain; sbellmunt@vhebron.net

16 Surgery Department, Universitat Autònoma de Barcelona, 08193 Barcelona, Spain

17 Vall d'Hebron Institut de Recerca, 08035 Barcelona, Spain

18 Internal Medicine Department, Germans Trias i Pujol University Hospital, 08916 Badalona, Spain

19 Pulmonology Department, Ramón y Cajal Hospital (IRYCIS), 28034 Madrid, Spain

20 Department of Medicine, Alcala University, 28805 Madrid, Spain

* Correspondence: ariera@bellvitgehospital.cat; Tel.: +34-93-260-76-99

$+\quad$ The COVILAX Project is explained in materials and methods.

Abstract: Patients with coronavirus disease 2019 (COVID-19) have a higher risk of venous thromboembolic disease (VTE) than patients with other infectious or inflammatory diseases, both as macrothrombosis (pulmonar embolism and deep vein thrombosis) or microthrombosis. However, the use of anticoagulation in this scenario remains controversial. This is a project that used DELPHI methodology to answer PICO questions related to anticoagulation in patients with COVID-19. The objective was to reach a consensus among multidisciplinary VTE experts providing answers to those PICO questions. Seven PICO questions regarding patients with COVID-19 responded with a broad consensus: 1 . It is recommended to avoid pharmacological thromboprophylaxis in most COVID-19 patients not requiring hospital admission; 2. In most hospitalized patients for COVID-19 who are receiving oral anticoagulants before admission, it is recommended to replace them by low molecular weight heparin (LMWH) at therapeutic doses; 3. Thromboprophylaxis with LMWH at standard doses is suggested for COVID-19 patients admitted to a conventional hospital ward; 
4. Standard-doses thromboprophylaxis with LMWH is recommended for COVID-19 patients requiring admission to Intensive Care Unit; 5 . It is recommended not to determine D-Dimer levels routinely in COVID-19 hospitalized patients to select those in whom VTE should be suspected, or as a part of the diagnostic algorithm to rule out or confirm a VTE event; 6 . It is recommended to discontinue pharmacological thromboprophylaxis at discharge in most patients hospitalized for COVID-19; 7 . It is recommended to withdraw anticoagulant treatment after 3 months in most patients with a VTE event associated with COVID-19. The combination of PICO questions and DELPHI methodology provides a consensus on different recommendations for anticoagulation management in patients with COVID-19.

Keywords: venous thromboembolic disease; COVID-19; coronavirus; pulmonary embolism; deep vein thrombosis; anticoagulation

\section{Introduction}

Coronavirus disease 2019 (COVID-19) mainly affects the respiratory system but is frequently accompanied by extrapulmonary manifestations [1,2]. There is strong evidence that patients with COVID-19 have a higher risk of arterial thrombosis and venous thromboembolic disease (VTE) than patients with other infectious or inflammatory diseases [3-5]. This increased risk of VTE, both in the form of deep vein thrombosis (DVT) and pulmonary thromboembolism (PTE), is due to the presence of hypercoagulability, endothelial damage, and venous stasis $[2,3,5]$. In addition, in some patients with COVID-19, pulmonary inflammation and local endothelial damage with subsequent complement system activation, thrombin generation, platelet and leukocyte recruitment, and the initiation of innate and adaptive immune responses, may lead to in situ pulmonary microthrombosis (thromboinflammation) $[3,6]$.

Although there are numerous publications on the prevention and treatment of VTE in patients with COVID-19, the evidence generated to date has not been sufficiently consistent to make solid recommendations [7-10]. For this reason, the aim of the present study was to seek a consensus among VTE experts on the use of anticoagulation in patients with COVID-19.

\section{Materials and Methods}

The COVILAX project (COVID-19 and thromboprophyLAXis of VTE) is a multidisciplinary consensus among experts in the management of patients with VTE. The aim was to improve knowledge of COVID-19-associated VTE and to provide clinicians with consensus recommendations in those areas that are more controversial and have less scientific evidence in prevention, diagnosis, and treatment. The study was approved by the Clinical Research Ethics Committee of the Hospital Universitari de Bellvitge (Barcelona, Spain; ethic approval number PR361/21).

The duration of the project was 6 months, between March and August 2021. The main objective was to reach a consensus on thromboprophylaxis, diagnosis, and treatment of VTE in patients with COVID-19 in different clinical situations, especially regarding the use of anticoagulant drugs. The methodology used established a set of clinical issues using PICO (Patient, Intervention, Comparison, Outcomes) questions and then applied DELPHI method to provide responses [11,12]. DELPHI is a structured methodology that systematically collects expert opinions on a particular issue and builds a general group consensus [13]. The benefits of the DELPHI methodology have been widely described in the literature [11-13]. A panel of 13 national experts was recruited for the project, which was developed in four phases. In phase 1, a group of experts (ARM, RL, SJ, MFV, MM, and DJ) defined the domains and PICO questions to be addressed. In phase 2, another group of experts (LJP, JTS, EG, ABM, APC, MPF, and SBM) discussed and answered the PICO questions. In phase 3 , the first group discussed the answers elaborated by the second group 
and after several rounds of feedback, drafted a response for each PICO question. In phase 4, each member of the panel individually expressed his/her agreement or disagreement with the answers to the PICO questions, and the degree of agreement was recorded. Throughout the project there was an active discussion and continuous feedback via the WITHIN3 platform (https: / / www.within3.com/), with individual password access.

The panel used the terms "recommended" or "suggested", if the strength of the recommendation was strong or weak, respectively [14]. The term standard-doses of pharmacological thromboprophylaxis refers to the usual doses of the different low-molecular-weight heparins (LMWH) for thromboprophylaxis according to their approved drug labeling, to differentiate them from intermediate or therapeutic anticoagulant doses.

\section{Results}

Four domains and seven PICO questions were defined (Table 1). Overall, there was an initial consensus on two general recommendations that affect all PICO questions. Firstly, a dose adjustment of LMWH is recommended in obese patients and in patients with renal insufficiency. In the case of patients with obesity (defined by a body mass index $>30 \mathrm{~kg} / \mathrm{m}^{2}$ or weight $>100 \mathrm{~kg}$ ), the adjustment would be made according to different recommendations [15-17]. In patients with renal failure (defined by a glomerular filtration rate $<30 \mathrm{~mL} / \mathrm{min}$ ), the recommended dose in each medication data sheet of the different LMWHs should be used [18]. Secondly, it is recommended to consider risk factors for bleeding (Table 2) and to assess bleeding risk individually in all patients with COVID-19 before initiating any anticoagulant therapy, although no agreement was reached on how this risk should be graded.

Table 1. Domains to be addressed with their respective PICO questions.

\begin{tabular}{|c|c|}
\hline Domain & PICO Question \\
\hline $\begin{array}{l}\text { Domain 1: thromboprophylaxis in } \\
\text { COVID-19 patients not requiring hospital } \\
\text { admission. }\end{array}$ & $\begin{array}{l}\text { PICO 1: Which patients with COVID-19 not requiring hospital admission should } \\
\text { receive standard-doses of pharmacological thromboprophylaxis? } \\
\text { P: COVID-19 patient without hospital admission criteria } \\
\text { I: standard-doses of pharmacological thromboprophylaxis } \\
\text { C: recommend mobilization (avoid sedentarism) but without pharmacological } \\
\text { thromboprophylaxis } \\
\text { O: symptomatic VTE }\end{array}$ \\
\hline \multirow{3}{*}{$\begin{array}{l}\text { Domain 2: thromboprophylaxis in hospi- } \\
\text { talized COVID-19 patients. }\end{array}$} & $\begin{array}{l}\text { PICO 2: In hospitalized patients for COVID-19 who were receiving } \\
\text { anticoagulation with oral anticoagulants prior to admission, should we substitute } \\
\text { them with LMWH? } \\
\text { P: COVID-19 patients admitted in a conventional ward who received previous oral } \\
\text { anticoagulant treatment } \\
\text { I: LMWH at anticoagulant dose } \\
\text { C: the same oral anticoagulant treatment prior to admission } \\
\text { O: the same goal of oral anticoagulation (prevent arterial or venous thrombosis) }\end{array}$ \\
\hline & $\begin{array}{l}\text { PICO 3: What is the optimal dose of LMWH for most COVID-19 patients admitted } \\
\text { to a conventional hospital ward? } \\
\text { P: COVID-19 patients admitted to a conventional hospital ward } \\
\text { I: intermediate or therapeutic doses of LMWH } \\
\text { C: standard doses of pharmacological thromboprophylaxis } \\
\text { O: symptomatic VTE/need for NIMV-IOT-ECMO/mortality/major bleeding }\end{array}$ \\
\hline & $\begin{array}{l}\text { PICO 4: What is the optimal dose of LMWH for most COVID-19 patients admitted } \\
\text { to an Intensive Care Unit (ICU)? } \\
\text { P: COVID-19 patients admitted to an ICU } \\
\text { I: intermediate or therapeutic doses of LMWH } \\
\text { C: standard-doses of pharmacological thromboprophylaxis } \\
\text { O: symptomatic VTE/need for NIMV-IOT-ECMO/mortality/major bleeding }\end{array}$ \\
\hline
\end{tabular}


Table 1. Cont.

\begin{tabular}{ll}
\hline Domain & PICO Question \\
\hline & PICO 5: Which is the significance of high D-dimer levels in hospitalized patients \\
& for COVID-19? \\
& P: patients admitted for COVID-19 \\
Domain 3: diagnosis and treatment guide & I: does the D-dimer have other utilities (diagnostic or therapeutic) in this context? \\
of vte in COVID-19 hospitalized patients. & C: continue using D-dimer in the context of low clinical suspicion due to its high \\
& negative predictive value \\
& O: symptomatic VTE/COVID-19 severity/mortality
\end{tabular}

PICO 6. Which patients without COVID-19-associated VTE should receive extended standard-doses thromboprophylaxis after hospital discharge?

P: patients without VTE during hospitalization for COVID-19 at hospital discharge I: extended thromboprophylaxis after discharge

C: thromboprophylaxis during hospitalization

Domain 4: follow-up after discharge of patients admitted for COVID-19 with and without a vte event.

O: symptomatic VTE/major bleeding/mortality

\section{PICO 7. Which is the optimal duration of anticoagulant treatment for patients} with COVID-19 associated VTE?

P: patient suffering VTE (proximal DVT and/or PE) associated with COVID-19

I: extended anticoagulant treatment (beyond the first 3 months)

$\mathrm{C}$ : anticoagulant treatment for 3 months

O: symptomatic recurrent VTE/major bleeding/clinically relevant non-major bleeding/mortality

Abbreviations. COVID-19: coronavirus disease 2019; PICO: Patient, Intervention, Comparison, Outcomes; VTE: venous thromboembolic disease; LMWH: low molecular weight heparins; NIMV-IOT-ECMO: non-invasive mechanical ventilation-orotracheal intubationextracorporeal membrane oxygenation; ICU: Intensive Care Units.

Table 2. Risk factors for bleeding with anticoagulant therapy.

\begin{tabular}{|c|}
\hline Advanced age (particularly $>75$ years) \\
\hline Previous bleeding (if not associated with a reversible or treatable cause) \\
\hline Recent surgery \\
\hline Active cancer \\
\hline Anaemia \\
\hline Thrombocytopenia (particularly $<50,000 / \mu \mathrm{L}$ ) \\
\hline Previous stroke, either haemorrhagic or ischaemic \\
\hline Renal failure \\
\hline Liver failure \\
\hline
\end{tabular}

3.1. Domain 1: Thromboprophylaxis in COVID-19 Patients Not Requiring Hospital Admission PICO 1: Which Patients with COVID-19 Not Requiring Hospital Admission Should Receive Standard-Doses of Pharmacological Thromboprophylaxis?

1. It is recommended to avoid pharmacological thromboprophylaxis in most COVID19 patients that do not require hospital admission.

2. It is suggested that standard-doses of thromboprophylaxis might be administered to those patients with symptomatic COVID-19 and risk factors for VTE (Table 3), although not requiring hospital admission. 
Table 3. Risk factors for venous thromboembolic disease.

\begin{tabular}{|c|}
\hline Obesity \\
\hline Family or personal history of VTE \\
\hline Active cancer \\
\hline Pregnancy/Puerperium/Estrogen replacement therapies \\
\hline $\begin{array}{l}\text { Recent history ( }<1 \text { month) of: Major surgery } \\
\text { Immobilization }(\geq 3 \text { days }) \text { due to acute medical illness }\end{array}$ \\
\hline $\begin{array}{l}\text { Hereditary thrombophilia (factor V Leiden, prothrombin G20210A mutation, protein C, S or } \\
\text { antithrombin III deficiencies) or acquired (antiphospholipid syndrome). }\end{array}$ \\
\hline $\begin{array}{l}\text { Consensus: } 13 / 13 \\
\text { Comments: There are no strong data to support routine use of standard-doses of } \\
\text { pharmacological thromboprophylaxis in these patients. A randomized clinical trial (RCT) } \\
\text { was published evaluating the efficacy and safety of sulodexide in patients with COVID-19 } \\
\text { who did not require hospital admission [19]. In this RCT, sulodexide significantly reduced } \\
\text { the need for hospital admission compared to placebo (17.7\% versus } 29.4 \%) \text { at } 21 \text { days. Some } \\
\text { major limitations of this trial are the lack of double-blinding, the small sample size, and the } \\
\text { heterogeneity of the concomitant treatments received by both groups. Pending the results } \\
\text { of ongoing RCTs (ClinicalTrials.gov: NCT04400799, NCT04492254), the panel considered } \\
\text { the increased risk of VTE in COVID-19 patients who have additional thrombotic risk } \\
\text { factors (Table 3), even if not requiring hospital admission, and suggests pharmacological } \\
\text { thromboprophylaxis when any of these factors coexist [2,20]. }\end{array}$ \\
\hline
\end{tabular}

\subsection{Domain 2: Thromboprophylaxis in Hospitalized COVID-19 Patients}

3.2.1. PICO 2: In Hospitalized Patients for COVID-19 Who Were Receiving Anticoagulation with Oral Anticoagulants Prior to Admission, Should We Substitute Them with LMWH?

1. In most hospitalized patients for COVID-19 who were receiving oral anticoagulants before admission, it is recommended to replace them by LMWH at therapeutic doses.

2. It is suggested to maintain treatment with vitamin $\mathrm{K}$ antagonists (VKA) in patients with mechanical heart valves who require hospitalization for COVID-19, if clinically stable.

Consensus: $13 / 13$

Comments: Patients with COVID-19 who require hospital admission receive treatments that could modify the effect of oral anticoagulants, mainly VKA [21]. In addition, these patients often require invasive procedures and may have difficulties with oral tolerance [22,23]. The use of LMWH during hospital admission minimizes the risk of drug-drug interactions and facilitates the performance of invasive procedures $[7,8]$. However, the use of VKAs is the recommended treatment for patients with mechanical heart valves. There are no data to support the use of non-vitamin $\mathrm{K}$ oral anticoagulants in these patients. The interruption of VKAs for planned invasive procedures or hospital admission for other reasons, such as COVID-19 infection, is a complex issue associated with multiple factors including the type and location of the prosthetic valve, reason for interruption, thromboembolic risk, and the duration of interruption. For clinically unstable patients, therapeutic bridging with either UFH or LMWH is required, although the evidence is scarce and a case-by-case evaluation should be performed [24].

3.2.2. PICO 3: What Is the Optimal Dose of LMWH for Most COVID-19 Patients Admitted to a Conventional Hospital Ward?

1. Thromboprophylaxis with LMWH at standard doses (versus therapeutic anticoagulation) is suggested for COVID-19 patients admitted to a conventional hospital ward, unless contraindicated.

Consensus: $13 / 13$

Comments: The main objectives of the different RCTs that analyze the possible benefits of therapeutic anticoagulation are not focused on VTE prevention but on COVID-19 
treatment $[22,25]$. In the ACTION RCT, which enrolled stable (more than 93\%) and unstable hospitalized patients with COVID-19, therapeutic anticoagulation with rivaroxaban 20 $\mathrm{mg} /$ day (in clinically stable patients) or enoxaparin $1 \mathrm{mg} / \mathrm{kg} / 12 \mathrm{~h}$ (in clinically unstable patients) was not superior to standard doses of thromboprophylaxis with enoxaparin in terms of the hierarchical composite endpoint of mortality, hospital stay, or days free from requiring oxygen therapy [22]. A recent multi-platform RCT (mpRCT) compared therapeutic-dose anticoagulation with LMWH with standard-dose thromboprophylaxis with LMWH in non-critically patients with COVID-19. The results showed an adjusted absolute difference in organ support, with free days up to day 21 among patients who survived to hospital discharge of $4 \%$ in favor of therapeutic-dose anticoagulation (95\% credible interval, 0.5-7.2) [25]. During hospitalization, 16 (1.4\%) venous thrombotic events occurred in patients receiving therapeutic anticoagulation and $26(2.5 \%)$ in those receiving standard-dose thromboprophylaxis. A major bleeding episode occurred in $1.9 \%(22 / 1180)$ of patients receiving therapeutic anticoagulation (three of them fatal), and in $0.9 \%(9 / 1047)$ of those receiving standard-dose thromboprophylaxis (one of them fatal). The open-label design, the different definitions used for critical and non-critical patients in the three platforms, the lack of detailed screening data to compare the most common reasons for exclusion from the trial, and the potential for ascertainment bias of major bleeding episodes are the main limitations of this study. Preliminary results of the RAPID RCT did not demonstrate a benefit of therapeutic anticoagulation with heparin compared to standard-dose thromboprophylaxis in patients admitted to a conventional hospital ward for COVID-19 and elevated D-dimer in terms of the combined primary endpoint of ICU admission or mechanical ventilation (invasive or non-invasive) or death at 28 days (16.2\% vs. $21.9 \%$ ). In the same RCT, the group receiving therapeutic anticoagulation had lower mortality than the group receiving standard-dose thromboprophylaxis (1.8\% vs. $7.6 \%)$. Surprisingly, the risk of bleeding was lower, although not statistically significant, in the group that received therapeutic anticoagulation than in the group that received standard doses of thromboprophylaxis ( $0.9 \%$ vs. $1.7 \%$ ) [26]. However, this RCT resulted as underpowered because at $75 \%$ of the originally planned sample size an interim analyses was pre-specified and established that the sample should be increased if the conditional power was between $60 \%$ and $80 \%$. Although the conditional power obtained was below $60 \%$, the sample size was not increased, thus RAPID trial remained underpowered for the analysis of the primary outcome. Moreover, when the authors performed a meta-analysis combining the results of the mpRCT in non-critically ill patients, the statistically significant reduction in overall mortality disappeared (6.4\% vs. $8.1 \%)$ and the risk of bleeding was higher in those who received therapeutic anticoagulation, although not statistically significant $(1.7 \%$ vs. $1.0 \%)[25,26]$. The HEP-COVID is a multicenter, pragmatic, pseudo-blinded RCT to evaluate efficacy and safety of therapeutic anticoagulant doses compared with intermediate/standard-doses thromboprophylaxis of LMWH in hospitalized COVID-19 patients with requirement for supplemental oxygen and a D-dimer $>4 \times$ upper limit of normal or sepsis-induced coagulopathy (SIC) score $\geq 4$. The primary composite endpoint was VTE, arterial thromboembolic events, and all-cause mortality at 30 days. Preliminary results in non-ICU patients randomized to therapeutic LMWH doses $(n=84)$ showed a statistically significant reduction of the primary endpoint compared to intermediate/standard doses of thromboprophylaxis of LMWH $(n=86)(16.7 \%$ vs. $36.1 \%)$. The risks of major bleeding in these non-ICU patients were similar (2.38\% vs. $2.33 \%$ ) [27].

3.2.3. PICO 4: What Is the Optimal Dose of LMWH for Most COVID-19 Patients Admitted to an Intensive Care Unit (ICU)?

1. Standard-doses thromboprophylaxis with LMWH (versus intermediate doses or therapeutic anticoagulation) is recommended for COVID-19 patients requiring admission to an ICU, unless there is contraindication.

2. A high suspicion index of VTE is recommended in COVID-19 patients admitted to ICU, particularly when there is clinical worsening with no evident alternative diagnosis.

Consensus: $12 / 13$ 
Comments: In the INSPIRATION RCT, patients admitted to ICU for COVID-19 were openly randomized to receive intermediate-dose enoxaparin $(1 \mathrm{mg} / \mathrm{kg} / \mathrm{d})$ or standard-dose thromboprophylaxis $(40 \mathrm{mg} / \mathrm{d}$ ). Intermediate dose administration did not significantly reduce the primary efficacy event, which was a combination of arterial or venous thrombosis, need for extracorporeal membrane oxygenation (ECMO), or 30-day mortality $(45.7 \%$ vs. $44.1 \%)$, and caused more major bleeding episodes ( $2.5 \%$ vs. $1.4 \%$ ) [23]. The mpRCT for critically ill patients with COVID-19 was stopped early due to futility in the primary outcome of organ support, with free days up to day 21 among patients who survived to hospital discharge. Moreover, the results showed no statistically significant differences between therapeutic anticoagulation or standard-dose thromboprophylaxis in survival $(62.7 \%$ vs. $64.5 \%)$ and there were more major bleeding events in patients that received therapeutic anticoagulation (3.8\% vs. $2.3 \%$ ) [28]. Regarding the use of intermediate doses, a recent RCT compared the use of standard-doses thromboprophylaxis with intermediate doses of enoxaparin in patients with COVID-19 admitted to ICU and/or had laboratory evidence of coagulopathy, defined by a modified International Society on Thrombosis and Haemostasis (ISTH) with an overt disseminated intravascular coagulation (DIC) score $\geq 3$. This RCT also found no significant differences in the primary endpoint of 30-day overall mortality (21\% vs. $15 \%$ ) [29]. Preliminary results of the HEP-COVID RCT in ICU patients showed no benefit in the primary composite endpoint (VTE, arterial thromboembolic events and all-cause mortality at 30 days) for those randomized to therapeutic LMWH doses $(n=45)$ compared to intermediate/standard-doses thromboprophylaxis of LMWH $(n=38)(51.1 \%$ vs. $55.3 \%)$ and higher risk of bleeding $(8.9 \%$ vs. $0 \%)$ [27].

In critically ill patients with COVID-19, it is not always possible to perform the usual diagnostic imaging tests (chest CT angiography, compression echography of the LMII, echocardiography), or the results are inconclusive when VTE is suspected. In this scenario, anticoagulation could be considered without diagnostic confirmation, once the patient's bleeding risk assessed is low [30].

\subsection{Domain 3: Diagnosis and Treatment Guide of VTE in COVID-19 Hospitalized Patients} PICO 5: Which Is the Significance of High D-Dimer Levels in Hospitalized Patients for COVID-19?

1. It is recommended not to determine D-Dimer levels routinely in COVID-19 hospitalized patients as a strategy to select those in whom VTE should be suspected, or as a part of the diagnostic algorithm for clinical suspicion to rule out or confirm a VTE event.

2. It is recommended not to use D-dimer levels to select the intensity of thromboprophylaxis with LMWH in patients requiring admission for COVID-19.

Consensus: $13 / 13$

Comments: In patients with COVID-19, elevated D-dimer levels are the result of the patient's inflammatory state and hypercoagulability [2,31]. High D-dimer levels have been associated with more severe forms of the disease and with a higher risk of bleeding, VTE, and mortality [2,30,32-35]. However, in these cases, D-dimer is not a screening test for VTE (to identify those in whom VTE should be suspected). In addition, most patients with COVID-19 have elevated D-dimer levels, therefore, its usefulness in ruling out VTE associated with COVID-19 without the need for imaging tests is reduced [2,4,33,34]. Moreover, there is no evidence that isolated levels of D-dimer justify a change in antithrombotic therapy. In fact, the effect of anticoagulation in both critically and noncritically ill patients, according to the results of the corresponding $\mathrm{mpRCT}$, was not influenced by stratification according to D-dimer levels $[25,28]$. Although cut-off levels with better sensitivity and specificity were described in a retrospective series of patients, a unanimous diagnostic cut-off point for VTE has not been defined [2,31-35]. However, VTE suspicion should arise in patients with clinical deterioration associated with high D-dimer levels and no elevation of other inflammatory markers. 
3.4. Domain 4: Follow-Up after Discharge of Patients Admitted for COVID-19 with and without a Vte Event

3.4.1. PICO 6. Which Patients without COVID-19-Associated VTE Should Receive Extended Standard-Doses Thromboprophylaxis after Hospital Discharge?

1. It is recommended to discontinue pharmacological thromboprophylaxis at discharge in most patients hospitalized for COVID-19, after a minimum of 6 days.

2. Extended duration of thromboprophylaxis with standard-doses of LMWH is suggested after hospital discharge to those patients with risk factors for VTE (Table 3).

Consensus: $12 / 13$

Comments: There are no strong data to support the use of extended thromboprophylaxis after hospital discharge in these patients. However, it is well known that the risk of VTE and mortality from PE remains high in the period following discharge from acute medical illness $[7,8]$. The use of extended thromboprophylaxis in medical patients was evaluated in five RCTs. One compared rivaroxaban $10 \mathrm{mg} /$ day versus placebo and the other four compared extended regimens (up to 42 days) of enoxaparin $40 \mathrm{mg} /$ day, apixaban $2.5 \mathrm{mg} / 12 \mathrm{~h}$, rivaroxaban $10 \mathrm{mg} /$ day, and betrixaban $80 \mathrm{mg} /$ day, versus enoxaparin $40 \mathrm{mg} /$ day for a minimum of 6-14 days [36]. A meta-analysis including these five RCTs concluded that extended thromboprophylaxis reduces symptomatic VTE but increases the risk of bleeding [36]. While some RCT results are pending (ClinicalTrials.gov: NCT046500871, NCT04662684), the panel took into account the increased risk of VTE in patients with COVID-19 who present other additional thrombotic risk factors (Table 3) and suggest extending thromboprophylaxis at discharge when any of these factors coexist $[2,20,36]$.

3.4.2. PICO 7. Which Is the Optimal Duration of Anticoagulant Treatment for Patients with COVID-19-Associated VTE?

1. It is recommended to withdraw anticoagulant treatment after 3 months in most patients with a VTE event associated with COVID-19.

2. It is suggested that the extension of anticoagulant treatment beyond the first 3 months should be evaluated in patients with respiratory symptoms associated with persistent COVID-19, particularly if there is the presence of persistently elevated inflammatory markers.

Consensus: $13 / 13$

Comments: The risk of recurrent VTE after anticoagulant therapy withdrawal is low in patients with a transient major risk factor, as is the case of COVID-19 hospitalization [7,8]. For this reason, a duration of anticoagulant treatment of 3 months is recommended for these patients $[7,8]$. Durations of less than 3 months have been associated with an increased risk of VTE recurrence, and longer durations have been associated with an increased risk of bleeding $[7,8,37]$. As in other clinical situations, it is recommended to extend anticoagulant treatment if the triggering risk factor is maintained $[7,8]$. Therefore, extended anticoagulation beyond 3 months could be considered for those patients with COVID-19associated VTE and respiratory symptoms related to persistent COVID-19 during follow-up, particularly in the presence of elevated inflammatory markers $[1,2,31]$.

\section{Discussion}

The pathophysiology of VTE development associated with COVID-19 infection is currently being unraveled. There is strong evidence that endothelial damage generated by SARS-CoV-2 Coronavirus (Severe Acute Respiratory Syndrome Coronavirus-2) is one of the main reasons for the excess of thrombotic events associated with COVID-19, compared to other infectious or inflammatory diseases. The direct effects of SARS-CoV-2 on pneumocytes and endothelium and the interaction between the systemic inflammatory response with the hemostatic system have been deemed the linchpin of the hypercoagulable state in COVID-19. In less than $20 \%$ of infected patients, a poorly controlled viral replication leads to apoptosis of pneumocytes and endothelial cells, which activate platelets and induce coagulation factors, increase cytokines production, and activate the complement system. 
The so-called cytokine storm fuels these proinflammatory and procoagulatory responses further, resulting in systemic endotheliitis, capillary leakage, endothelial and organ dysfunction, and overt activation of the coagulation cascade that leads to a high incidence of microthrombi and macrothrombi and the subsequent need for organ support [1-4]. This thrombotic state is the result of a process known as immunothrombosis, in which the immune and coagulation systems cooperate to block pathogens, such as SARS-CoV-2, and limit their spread. In COVID-19 infection, a deregulated and exaggerated process of immunothrombosis, mainly involving the pulmonary microcirculation, drives the severe clinical manifestations of COVID-19 [38].

However, how to control the hypercoagulable state generated by this endothelial dysfunction remains controversial. Regarding the use of anticoagulation with heparin at therapeutic doses, all the RCTs carried out in critically ill patients have obtained similar results, with no evidence of clinical benefit [23,27-29]. Therapeutic anticoagulation may be unable to influence the inflammatory cascade and extensive pulmonary microthrombosis secondary to severe endothelial damage, which is characteristic of these critically ill patients $[2,3]$.

It remains to be confirmed whether early administration of therapeutic anticoagulation in non-critically ill patients prevents progression to this extensive endothelial damage and pulmonary microthrombosis and improves prognosis. In addition to an optimal route of administration for the COVID-19 patient profile, the pleiotropic (anti-inflammatory or antiviral) effects of LMWH may provide additional benefits over oral anticoagulants $[22,39]$. These pleiotropic effects could have influenced the benefits obtained with higher doses of LMWH obtained in the non-critically ill patient mpRCT, where only approximately $60 \%$ of patients received corticotherapy [25]. In addition, the limitations inherent to the design of the mpRCTs should be highlighted, where the control group is not concurrent with the experimental group and the patients enter and exit the trial at different times [40]. It should also be considered that in the non-critically ill patient $\mathrm{mpRCT}, 20.4 \%$ of the anticoagulation group did not receive therapeutic doses and $26.5 \%$ of the control group received intermediate doses of LMWH [25]. Moreover, the distinct definitions for the VTE outcomes used in the different RCTs could explain the divergent results among the RCTs, mainly in non-critically ill patients [25-27]. Due to these limitations and those previously mentioned, which generate uncertainty regarding the possible benefit of therapeutic anticoagulant doses for hospitalized patients in conventional wards, further studies are needed to state more solid recommendations [25-27].

In addition to these limitations, the risk of bleeding should be assessed on an individualized and continuous basis, especially if higher rather than standard thromboprophylaxis doses are administered, since they are associated with a higher incidence of bleeding [41,42]. A meta-analysis involving 7781 patients hospitalized for COVID-19 showed that standard doses of thromboprophylaxis were not associated with an excess of bleeding compared to no thromboprophylaxis, while the use of therapeutic anticoagulation was associated with more than two-fold increased risk of bleeding than standard thromboprophylaxis [41]. This excess of bleeding events needs to be weighed against any potential benefit of therapeutic anticoagulant doses as a part of COVID-19 treatment. Moreover, a recent study reported that patients developing VTE after COVID-19 infection are at a higher risk for major bleeding than VTE recurrences during the first 3 months of therapy [43]. Since patients with COVID-19 infection are at an increased risk for both VTE and bleeding, the management of VTE should be always tailored to their clinical and biological characteristics. For hospitalized patients for COVID-19 with a high bleeding risk, mechanical prophylaxis should be instituted and continued until standard-doses of pharmacological thromboprophylaxis can be initiated. For hemodynamically stable COVID-19 patients with an acute VTE event and contraindication for anticoagulant treatment, an inferior vena cava filter should be placed to prevent PE. High-risk PE patients with COVID-19 and high bleeding risk require immediate reperfusion with percutaneous techniques or surgical embolectomy. In all these 
scenarios, clinical decisions should be based on an individual assessment of the thrombosis and bleeding risks, according to international guidelines for VTE management $[7,8]$.

This study has some limitations. First, the selection of experts was arbitrary [11]. However, they are specialists with extensive experience in the field of VTE in different settings (primary care, hospitalization, and ICU). Second, the limitations of the DELPHI methodology have been described previously [13]. Despite this, the consensus criteria were defined a priori and the majority agreement on the seven PICO questions supports the consistency of the recommendations and suggestions. Finally, the scientific evidence in this field is evolving rapidly, so current recommendations may be modified according to the results of new RCTs.

In conclusion, the combination of PICO questions and DELPHI methodology provides a consensus on relevant and controversial issues about the use of anticoagulation in patients with COVID-19.

Author Contributions: Conceptualization, A.R.-M.; methodology and supervision, A.R.-M., M.M. and D.J.; data curation, investigation, validation, writing —original draft, writing-review and editing, all authors. All authors have read and agreed to the published version of the manuscript.

Funding: This work was carried out with the institutional support and unconditional financial assistance of Sanofi, which had no role in the design, interpretation, or writing of the manuscript.

Conflicts of Interest: Riera-Mestre A: has received fees for presentations and non-financial support from Sanofi, Rovi, Techdow, Bayer Healthcare, BMS-Pfizer and Daichii Sanchyo. Jara-Palomares L: has received honoraria for presentations from Bayer Hispania, Actelion, Pfizer, Rovi, LEO Pharma, Daiichi Sankyo grants, personal fees, and non-financial support from LEO Pharma, Menarini, MSD, and Roche. Lecumberri R: consulting fees for Aspen and Leo-Pharma; honoraria for presentations from Bristol-Myers Squibb, Boehringer Ingelheim, Daiichi Sankyo, Leo Pharma, Laboratorios Rovi and Sanofi. Trujillo-Santos J: has received fees from Bayer, BMS, Pfizer, Boehringer-Ingelheim, DaiichiSankyo, Rovi, Sanofi, Leo-Pharma and Aspen, and fees for conferences from Bayer, BMS, Pfizer, Boehringer-Ingelheim, Daiichi-Sankyo, Rovi, Sanofi, Leo-Pharma, Aspen. Grau E: no current conflicts of interest. Blanco-Molina A: has received conference fees from Rovi, Sanofi, Pfizer, Bristol Myers Squib, Exeltis. Piera Carbonell A: has received fees for her participation in lectures, talks, courses, conferences, or publications with Novo Nordisk, Novartis, MSD, Ferrer, Bayer, Sanofi-Aventis, AstraZéneca, Boehringer-Ingelheim, Mylan. Jiménez S: has received honoraria for her participation in presentations at conferences sponsored by ROVI, Leo-Pharma, and Aspen. Frías Vargas, M: has received fees for grants, lectures, or consultancy work from Sanofi, Italfarmaco, Rovi and Viatris. Fuset MP: has received conference fees from Abbott. Bellmunt-Montoya S: has received fees from Sanofi, Bayer for advisory board and Rovi for conferences. Monreal M: unrestricted educational grant to sponsor the RIETE registry from Sanofi, Leo, and Rovi; honoraria for advisory meetings from Sanofi, Leo, and Alfa Sigma; fees for giving lectures or presentations from Sanofi, Leo, Alfa Sigma, and Pfizer. Jiménez D: has received conference fees from Bayer, Boehringer Ingelheim, Bristol-Myers Squibb, Daiichi-Sankyo, Leo-Pharma, Pfizer, Rovi, and Sanofi; consulting fees from Bayer, Boehringer Ingelheim, Bristol-Myers Squibb, Daiichi-Sankyo, Leo-Pharma, Pfizer, Rovi, and Sanofi; and research grants from GSK, Daiichi-Sankyo, Rovi, and Sanofi. The funders had no role in the design of the study; in the collection, analyses, or interpretation of data; in the writing of the manuscript; or in the decision to publish the results.

\section{References}

1. Siddiqi, H.K.; Mehra, M.R. COVID-19 illness in native and immunosuppressed states: A clinical-therapeutic staging proposal. J. Heart Lung Transplant. 2020, 39, 405-407. [CrossRef]

2. Leentjens, J.; van Haaps, T.F.; Wessels, P.F.; Schutgens, R.E.G.; Middeldorp, S. COVID-19-associated coagulopathy and antithrombotic agents-lessons after 1 year. Lancet Haematol. 2021, 8, e524-e533. [CrossRef]

3. Ackermann, M.; Verleden, S.; Kuehnel, M.; Haverich, A.; Welte, T.; Laenger, F.; Vanstapel, A.; Werlein, C.; Stark, H.; Tzankov, A.; et al. Pulmonary vascular endothelialitis, thrombosis, and angiogenesis in COVID-19. N. Engl. J. Med. 2020, 383, 120-128. [CrossRef] [PubMed] 
4. Helms, J.; CRICS TRIGGERSEP Group (Clinical Research in Intensive Care and Sepsis Trial Group for Global Evaluation and Research in Sepsis); Tacquard, C.; Severac, F.; Leonard-Lorant, I.; Ohana, M.; Delabranche, X.; Merdji, H.; Clere-Jehl, R.; Schenck, M.; et al. High risk of thrombosis in patients with severe SARS-CoV-2 infection: A multicenter prospective cohort study. Intensiv. Care Med. 2020, 46, 1089-1098. [CrossRef]

5. Li, J.; Wang, H.; Yin, P.; Li, D.; Wang, D.; Peng, P.; Wang, W.; Wang, L.; Yuan, X.; Xie, J.; et al. Clinical characteristics and risk factors for symptomatic venous thromboembolism in hospitalized COVID-19 patients: A multicenter retrospective study. J. Thromb. Haemost. 2021, 19, 1038-1048. [CrossRef]

6. Jackson, S.P.; Darbousset, R.; Schoenwaelder, S.M. Thromboinflammation: Challenges of therapeutically targeting coagulation and other host defense mechanisms. Blood 2019, 133, 906-918. [CrossRef]

7. Konstantinides, S.V.; Meyer, G.; Becattini, C.; Bueno, H.; Geersing, G.-J.; Harjola, V.-P.; Huisman, M.V.; Humbert, M.; Jennings, C.S.; Jiménez, D.; et al. 2019 ESC Guidelines for the diagnosis and management of acute pulmonary embolism developed in collaboration with the European Respiratory Society (ERS): The task force for the diagnosis and management of acute pulmonary embolism of the European Society of Cardiology (ESC). Eur. Respir. J. 2019, 54, 1901647. [CrossRef]

8. Kearon, C.; Akl, E.A.; Ornelas, J.; Blaivas, A.; Jimenez, D.; Bounameaux, H.; Huisman, M.; King, C.S.; Morris, T.A.; Sood, N.; et al. Antithrombotic therapy for VTE disease: CHEST guideline and expert panel report. Chest 2016, 149, 315-352. [CrossRef] [PubMed]

9. Patell, R.; Chiasakul, T.; Bauer, E.; Zwicker, J.I. Pharmacologic thromboprophylaxis and thrombosis in hospitalized patients with COVID-19: A pooled analysis. Thromb. Haemost. 2021, 121, 76-85. [CrossRef]

10. Carbonell, A.P.; Vargas, M.F.; Vallejo, O.G.; Lerín, A.G.; Ferriols, M.C.; Morant, J.P.; Carrasco, E.C.; en nombre del Grupo de trabajo de Vasculopatías de SEMERGEN. COVID-19 and thromboprophylaxis: Recommendations for our clinical practice in Primary Care. Semergen 2020, 46, 479-486. [CrossRef]

11. Blaschke, S.; Lambert, S.; Livingston, P.M.; Aranda, S.; Boltong, A.; Schofield, P.; Chambers, S.K.; Krishnasamy, M.; Ugalde, A. Identifying priorities for cancer caregiver interventions: Protocol for a three-round modified Delphi study. BMJ Open 2019, 9, e024725. [CrossRef] [PubMed]

12. Milinis, K.; Thapar, A.; Shalhoub, J.; Davies, A.H. Antithrombotic therapy following venous stenting: International delphi consensus. Eur. J. Vasc. Endovasc. Surg. 2018, 55, 537-544. [CrossRef] [PubMed]

13. Graham, B.; Regehr, G.; Wright, J.G. Delphi as a method to establish consensus for diagnostic criteria. J. Clin. Epidemiol. 2003, 56, 1150-1156. [CrossRef]

14. Andrews, J.; Guyatt, G.; Oxman, A.D.; Alderson, P.; Dahm, P.; Falck-Ytter, Y.; Nasser, M.; Meerpohl, J.; Post, P.N.; Kunz, R.; et al. GRADE guidelines: 14. Going from evidence to recommendations: The significance and presentation of recommendations. J. Clin. Epidemiol. 2013, 66, 719-725. [CrossRef] [PubMed]

15. Hirsh, J.; Guyatt, G.; Albers, G.W.; Harrington, R.; Schünemann, H.J. Antithrombotic and thrombolytic therapy, 8th Edition: American college of chest physicians evidence-based clinical practice guidelines. Chest 2008, 133, 110S-112S. [CrossRef]

16. Garcia, D.A.; Baglin, T.P.; Weitz, J.I.; Samama, M.M. Parenteral anticoagulants: Antithrombotic therapy and prevention of thrombosis, 9th ed: American college of chest physicians evidence-based clinical practice guidelines. Chest 2012, 141, e24S-e43S. [CrossRef]

17. Miranda, S.; Le Cam-Duchez, V.; Benichou, J.; Donnadieu, N.; Barbay, V.; Le Besnerais, M.; Delmas, F.-X.; Cuvelier, A.; Lévesque, H.; Benhamou, Y.; et al. Adjusted value of thromboprophylaxis in hospitalized obese patients: A comparative study of two regimens of enoxaparin: The ITOHENOX study. Thromb. Res. 2017, 155, 1-5. [CrossRef]

18. Agencia Española de Medicamentos y Productos Sanitarios. Available online: https://cima.aemps.es/cima/publico/home.html (accessed on 22 July 2021).

19. Ochoa, A.J.G.; Raffetto, J.D.; Hernández, A.G.; Zavala, N.; Gutiérrez, O.; Vargas, A.; Loustaunau, J. Sulodexide in the treatment of patients with early stages of COVID-19: A randomized controlled trial. Thromb. Haemost. 2021, 121, 944-954. [CrossRef]

20. Rosendaal, F.R. Venous thrombosis: A multicausal disease. Lancet 1999, 353, 1167-1173. [CrossRef]

21. Testa, S.; Paoletti, O.; Giorgi-Pierfranceschi, M.; Pan, A. Switch from oral anticoagulants to parenteral heparin in SARS-CoV-2 hospitalized patients. Intern. Emerg. Med. 2020, 15, 751-753. [CrossRef]

22. Lopes, R.D.; Silva, P.G.M.D.B.E.; Furtado, R.H.M.; Macedo, A.V.S.; Bronhara, B.; Damiani, L.P.; Barbosa, L.M.; Morata, J.d.A.; Ramacciotti, E.; Martins, P.D.A.; et al. Therapeutic versus prophylactic anticoagulation for patients admitted to hospital with COVID-19 and elevated D-dimer concentration (ACTION): An open-label, multicentre, randomised, controlled trial. Lancet 2021, 397, 2253-2263. [CrossRef]

23. INSPIRATION Investigators; Sadeghipour, P.; Talasaz, A.H.; Rashidi, F.; Sharif-Kashani, B.; Beigmohammadi, M.T.; Farrokhpour, M.; Sezavar, S.H.; Payandemehr, P.; Dabbagh, A.; et al. Effect of intermediate-dose vs standard-dose prophylactic anticoagulation on thrombotic events, extracorporeal membrane oxygenation treatment, or mortality among patients with COVID-19 admitted to the intensive care unit: The inspiration randomized clinical trial. JAMA 2021, 325, 1620-1630. [CrossRef]

24. Vahanian, A.; Beyersdorf, F.; Praz, F.; Milojevic, M.; Baldus, S.; Bauersachs, J.; Capodanno, D.; Conradi, L.; De Bonis, M.; De Paulis, R.; et al. ESC/EACTS Scientific Document Group. 2021 ESC/EACTS Guidelines for the management of valvular heart disease. Eur. Heart J. 2021, ehab395. [CrossRef]

25. The ATTACC, ACTIV-4a, and REMAP-CAP Investigators. Therapeutic anticoagulation with heparin in noncritically ill patients with COVID-19. N. Engl. J. Med. 2021, 385, 790-802. [CrossRef] 
26. Sholzberg, M.; Tang, G.H.; Rahhal, H.; AlHamzah, M.; Kreuziger, L.B.; Áinle, F.N.; Alomran, F.; Alayed, K.; Alsheef, M. The RAPID Trial Investigators; et al. Heparin for moderately Ill patients with COVID-19. medRxiv 2021.07.08.21259351. [CrossRef]

27. European Society of Cardiology Congress 2021. Systemic Anticoagulation with Full Dose LMWH vs. Prophylactic or Intermediate Dose Heparins in High Risk Hospitalized COVID-19 Patients (HEP-COVID) Trial. Primary Results. Available online: https: / / www.escardio.org/Congresses-\&-Events/ESC-Congress (accessed on 31 August 2021).

28. REMAP-CAP, ACTIV-4a, and ATTACC Investigators. Therapeutic anticoagulation with heparin in critically ill patients with COVID-19. N. Engl. J. Med. 2021, 385, 777-789. [CrossRef] [PubMed]

29. Perepu, U.S.; Chambers, I.; Wahab, A.; Eyck, P.T.; Wu, C.; Dayal, S.; Sutamtewagul, G.; Bailey, S.R.; Rosenstein, L.J.; Lentz, S.R. Standard prophylactic versus intermediate dose enoxaparin in adults with severe COVID-19: A multi-center, open-label, randomized controlled trial. J. Thromb. Haemost. 2021, 19, 2225-2234. [CrossRef]

30. Demelo-Rodriguez, P.; Farfán-Sedano, A.I.; Pedrajas, J.M.; Llamas, P.; Sigüenza, P.; Jaras, M.J.; Quintana-Diaz, M.; FernándezCapitán, C.; Bikdeli, B.; Jiménez, D.; et al. Bleeding risk in hospitalized patients with COVID-19 receiving intermediate- or therapeutic doses of thromboprophylaxis. J. Thromb. Haemost. 2021, 19, 1981-1989. [CrossRef] [PubMed]

31. Berkman, S.A.; Tapson, V.F. COVID-19 and its implications for thrombosis and anticoagulation. Semin. Respir. Crit. Care Med. 2021, 42, 316-326. [CrossRef] [PubMed]

32. García-Cervera, C.; Giner-Galvañ, V.; Wikman-Jorgensen, P.; Laureiro, J.; Rubio-Rivas, M.; Arena, A.G.; Arnalich-Fernandez, F.; Pérez, J.L.B.; Núñez, J.A.V.; Igual, J.J.G.; et al. SEMI-COVID-19 Network. Estimation of admission D-dimer cut-off value to predict venous thrombotic events in hospitalized COVID-19 patients: Analysis of the SEMI-COVID-19 registry. J. Gen. Intern. Med. 2021, 1-9. [CrossRef]

33. Zhan, H.; Chen, H.; Liu, C.; Cheng, L.; Yan, S.; Li, H.; Li, Y. Diagnostic value of D-dimer in COVID-19: A meta-analysis and meta-regression. Clin. Appl. Thromb. Hemost. 2021, 27, 10760296211010976. [CrossRef] [PubMed]

34. Cerdà, P.; Ribas, J.; Iriarte, A.; Mora-Luján, J.M.; Torres, R.; Del Río, B.; Jofre, H.I.; Ruiz, Y.; Huguet, M.; Fuset, M.P.; et al. Blood test dynamics in hospitalized COVID-19 patients: Potential utility of D-dimer for pulmonary embolism diagnosis. PLoS ONE 2020, 15, e0243533. [CrossRef]

35. Bellmunt-Montoya, S.; Riera, C.; Gil, D.; Rodríguez, M.; García-Reyes, M.; Martínez-Carnovale, L.; Marrero, C.; Gil, M.; RuizRodríguez, J.C.; Ferrer, R.; et al. COVID-19 infection in critically Ill patients carries a high risk of venous thrombo-embolism. Eur. J. Vasc. Endovasc. Surg. 2021, 61, 628-634. [CrossRef] [PubMed]

36. Zayed, Y.; Kheiri, B.; Barbarawi, M.; Banifadel, M.; Abdalla, A.; Chahine, A.; Obeid, M.; Haykal, T.; Yelangi, A.; Malapati, S.; et al. Extended duration of thromboprophylaxis for medically ill patients: A systematic review and meta-analysis of randomised controlled trials. Intern. Med. J. 2020, 50, 192-199. [CrossRef] [PubMed]

37. Boutitie, F.; Pinede, L.; Schulman, S.; Agnelli, G.; Raskob, G.; Julian, J.; Hirsh, J.; Kearon, C. Influence of preceding length of anticoagulant treatment and initial presentation of venous thromboembolism on risk of recurrence after stopping treatment: Analysis of individual participants' data from seven trials. BMJ 2011, 342, d3036. [CrossRef]

38. Bonaventura, A.; Vecchié, A.; Dagna, L.; Martinod, K.; Dixon, D.L.; Van Tassell, B.W.; Dentali, F.; Montecucco, F.; Massberg, S.; Levi, M.; et al. Endothelial dysfunction and immunothrombosis as key pathogenic mechanisms in COVID-19. Nat. Rev. Immunol. 2021, 21, 319-329. [CrossRef] [PubMed]

39. Buijsers, B.; Yanginlar, C.; Maciej-Hulme, M.L.; de Mast, Q.; van der Vlag, J. Beneficial non-anticoagulant mechanisms underlying heparin treatment of COVID-19 patients. EBioMedicine 2020, 59, 102969. [CrossRef]

40. Dodd, L.E.; Freidlin, B.; Korn, E.L. Platform trials-Beware the noncomparable control group. N. Engl. J. Med. 2021, 384, 1572-1573. [CrossRef]

41. Parisi, R.; Costanzo, S.; Di Castelnuovo, A.; de Gaetano, G.; Donati, M.B.; Iacoviello, L. Different anticoagulant regimens, mortality, and bleeding in hospitalized patients with COVID-19: A systematic review and an updated meta-analysis. Semin. Thromb. Hemost. 2021, 47, 372-391. [CrossRef]

42. Jiménez, D.; García-Sanchez, A.; Rali, P.; Muriel, A.; Bikdeli, B.; Ruiz-Artacho, P.; Le Mao, R.; Rodríguez, C.; Hunt, B.J.; Monreal, M. Incidence of VTE and bleeding among hospitalized patients with coronavirus disease 2019: A systematic review and meta-analysis. Chest 2021, 159, 1182-1196. [CrossRef]

43. Buso, G.; Mazzolai, L.; Rueda-Camino, J.A.; Fernández-Capitán, C.; Jiménez, D.; Bikdeli, B. Pulmonary embolism in patients with COVID-19: Comparison between different care settings. Semin. Thromb. Haemost. 2021, in press. 